Revista Multidisciplinar do Nordeste Mineiro, v.2 2019/02

ISSN 2178-6925

\title{
OS POSSÍVEIS ESTRESSES CAUSADOS EM ANIMAIS UTILIZADOS EM TERAPIAS: CINOTERAPIA E EQUOTERAPIA
}

\section{THE POSSIBLE STRESSES CAUSED IN ANIMALS USED IN THERAPIES: CYNOTHERAPY AND EQUOTHERAPY}

\begin{abstract}
Clara Barbosa da Costa
Discente do curso de Medicina Veterinária pelo Centro Universitário Doctum de Teófilo Otoni - Unidoctum.

E-mail: clarabarbosac@gmail.com
\end{abstract}

lanne Rodrigues Cordeiro

Discente do curso de Medicina Veterinária pelo Centro Universitário Doctum de Teófilo Otoni - Unidoctum. E-mail:iannecordeiro@hotmail.com

Juliana Nunes Ramos Vaz Discente do curso de Medicina Veterinária pelo Centro Universitário Doctum de Teófilo Otoni - Unidoctum.

E-mail:jununes11@live.com

Marcela Gonçalves Cangussu

Médica Veterinária

Mestre em Zootecnia-Produção Animal pela Universidade Federal de Minas Gerais. Graduada em Medicina Veterinária pela Universidade Vila Velha. Professora do curso de Medicina Veterinária pelo Centro Universitário Doctum de Teófilo Otoni e Professora do curso de Agronomia pela Faculdade Presidente Antônio Carlos de Teófilo Otoni E-mail: mvmarcelacangussu@yahoo.com.br

\section{Resumo}

Os benefícios originados da relação homem-animal são indicados por diversas literaturas, onde o animal assume um papel de terapeuta em prol da saúde humana, na denominada terapia assistida por animais, ainda que ele possa vir a passar por situações de estresse a partir dessa interação. Desse modo, o objetivo do trabalho foi identificar quais são esses estresses, bem como os motivos pelos quais eles são causados, mantendo o foco em caninos e equinos. Como método da condução do estudo, foi realizada uma pesquisa exploratória a qual propiciou uma revisão bibliográfica em torno no tema. A análise de dados indica que a relação do animal com o homem pode desencadear possíveis estresses nos animais em terapia, decorrentes dos treinos, da terapia emsi e de fatores externos, principalmente novos estímulos do ambiente. Os tipos de estresse sofridos são tanto físicos quanto emocionais, com uma duração indefinida nas literaturas. Apesar de ser mostrado que alguns agentes estressores contínuos podem conduzir ao estresse crônico. Para tanto, o animal selecionado para a terapia deve passar por uma série de treinamentos específicos, visando uma minimização do estresse na prática terapêutica. Concluiu-se assim que a garantia do bem-estar é essencial na terapia assistida por animais, que deve ser respaldada pela bioética e pelos direitos dos 
animais, a fim de evitar qualquer dor ou sofrimento a eles. A terapia assistida por animais e o bem-estar não podem ser excludentes um do outro, tendo em vista a consolidação de uma relação simétrica entre ambas as espécies.

Palavras-chave: Terapia assistida por animais; Bem-estar; Equoterapia; Cinoterapia;Estresse.

\section{Abstract}

The benefits derived from the man-animal relationship areindicated by several literatures, where the animal assumes a role of therapist in favor of human health, in the so-called animal-assisted therapy, although it may come under stress from this interaction. In this way, the objective of the work was to identify what these stresses are, as well as the reasons why they are caused, keeping the focus on canines and horses. As a method of conducting the study, an exploratory research was carried out which provided a bibliographic review around the theme. Data analysis indicates that the relationship between the animal and the man can trigger possible stresses in the animals in therapy, resulting from the training, the therapy itself and external factors, especially new environmental stimuli.The types of stress suffered are both physical and emotional, with an indefinite duration in literatures. Although it is shown that some continuous stressors can lead to chronic stress. To do so, the animal selected for the therapy must undergo a series of specific training, aiming at minimizing stress in therapeutic practice. It was thus concluded that the guarantee of well-being is essential in animal assisted therapy, which should be backed by bioethics and animal rights in order to avoid any pain or suffering to them. Animalassisted therapy and well-being can not be mutually exclusive, in view of the consolidation of a symmetrical relationship between the two species.

Keywords: Animal-assisted therapy; Well-being; Equine therapy; Cinoterapia; Stress.

\section{Introdução}

A terapia assistida por animais se baseia em critérios específicos, onde o animal é a parte principal do tratamento, objetivando promover a melhora social, emocional, física e/ou cognitiva de pacientes humanos (MACHADO, et al., 2008, apud FERREIRA; GOMES, 2017). Nos dias atuais essa prática é reconhecida em diversos países do mundo, sendo que foi utilizada pela primeira vez em aproximadamente 400 anos a.C., por Hipócrates, um grego que é declarado o pai da medicina. Ele observou que a interação de pessoas com o cavalo gerava benefícios neurológicos a elas, o que mais tarde veio a se tornar algo verídico.

A partir da revisão bibliográfica realizada para 0 presente trabalho, identificou-se que a terapia assistida por animais utiliza várias espécies diferentes para fins terapêuticos, como o cavalo, o cão, o gato, golfinho, pássaros, animais de 
fazenda e animais silvestres. A equoterapia e a cinoterapia são as modalidades mais utilizadas no Brasil. O contato com o cavalo é capaz de despertar e melhorar habilidades motoras do praticante, dentre outros benefícios. E o cão atua como instrumento reforçador, estimulador e reabilitador social e emocional dos pacientes.

Entretanto, esses animais devem passar por uma avaliação com médicos veterinários e com especialistas em comportamento animal, devendo ser avaliados em três aspectos (saúde, treinamento e comportamento/temperamento), por meio de testes que possam medir e averiguar a aptidão do animal para fazer parte da terapia, elucidando o bem-estar necessário e o estresse a ser evitado.

Preservar o direito desses animais terapeutas é fundamental, mantendo um olhar bioético para a participação deles em práticas terapêuticas, oferecendo condições ideais tanto no aspecto físico quanto no emocional. Ofertar afeto a esses animais é algo simples que pode fazer muita diferença na qualidade de vida deles.

Logo, o objetivo desse trabalho foi realizar um levantamento de informações por meio de diferentes autores e, a partir disso, apontar os possíveis estresses causados em animais utilizados em terapias, bem como os agentes causadores desses estresses, apontando alguns efeitos dos mesmos em equinos e caninos. Destacando assim a base moral, centrada no animal, em torno desse tipo de terapia.

\section{Terapia assistida por Animais}

A interação homem-animal data os antepassados da humanidade, onde o animal foi muito utilizado como auxilio no trabalho humano e para o consumo, sendo considerado primordial para o desenvolvimento e evolução da humanidade. Segundo Grandin e Jhonson (2010, apud FISCHER et al., 2016), essa exploração animal só foi possível devido à domesticação, decorrente de alterações genéticas espontâneas promotoras da adaptação ao homem e ao ambiente alterado.

Desse modo, domesticar o animal deu início a criação de um vínculo a partir da interação homem-animal, em que esses animais adquiriram gradualmente um papel muito maior na sociedade. Seu papel como auxiliador se expandiu nos mais diversos âmbitos, inclusive como beneficiador para a saúde humana, proporcionando nesse sentido o início da terapia assistida por animais (TAA).

Os primeiros registros da utilização de animais como beneficiadores da saúde humana ocorreram na Inglaterra, em 1792, quando Willian Tuke fez uso de animais 
de fazenda com o intuito de analisar os estímulos positivos que deficientes mentais poderiam obter a partir desse contato. Na época o método era conhecido como tratamento humanizado e, apesar dos resultados satisfatórios, estudos científicos sobre o tema só surgiram anos mais tarde.

No Brasil, a prática foi implementada pela Dra. Nise da Silveira, no hospital psiquiátrico Dom Pedro II, em 1946, no Rio de Janeiro. Mas o marco oficial se deu pela implementação do Projeto Pet Smile, pela psicóloga e veterinária Hannelore Fuchs em 1997, um serviço comunitário filantrópico (FISCHER et al., 2016).

A TAA é definida como um processo terapêutico adotado mundialmente e padronizado pela organização norte americana sem fins lucrativos, Society Delta, a qual desde 1997 fomenta a melhoria na saúde e qualidade de vida humana, através do auxílio dos animais (FERREIRA, 2012, apud FISCHER et al., 2016). Nela, o animal é o meio pelo qual o tratamento é feito, conferindo a terapia o objetivo de promover benefícios e auxiliar na recuperação social, emocional, física e/ou cognitiva de crianças e adultos (MACHADO et al., 2008, apud FERREIRA; GOMES, 2017).

Qualquer tipo de animal que possa entrar em contato com o homem, sem oferecer a ele um perigo controlado, pode ser usado para a terapia, desde que animal seja devidamente treinado. Levando em conta a variabilidade das espécies, a TAA se divide em diferentes modalidades, desenvolvidas de formas diferentes, de acordo com a necessidade de quem a recebe.

Para esse desenvolvimento é necessário o trabalho conjunto de uma equipe multidisciplinar que acompanhe as atividades terapêuticas e garanta o bem-estar dos animais e dos pacientes (ou praticantes, no caso da equoterapia). Outro ponto importante é que o animal seja credenciado, passando por uma série de treinamentos específicos antes de se tornar parte integrante em algum programa de TAA.

O certo é que os animais utilizados sejam isentos de qualquer sofrimento físico ou mental (SINGER, 2004, apud FISCHER et al., 2016), porque eles são seres sencientes que não tem interesse em passar por qualquer tipo de sofrimento.

Ainda há uma escassez de metodologia empírica quando se busca sobre o tema, o que mostra a necessidade de mais pesquisas relacionadas a ele, principalmente com foco no animal. Esses "atores principais" da técnica são totalmente beneficiados ao serem utilizados com fins terapêuticos? Há indícios de 
estresse nesse método? São questões que geram controvérsias entre diferentes autores, pois os benefícios para os humanos a partir da TAA são variados, como demonstrado em uma análise histórica e social feita por Capote (2009, apud FISCHER et al., 2016). Contudo, se há escassez sobre o tema em geral, são quase nulos os materiais que abordam esse tipo de terapia, com foco nos animais.

O que se sabe é que a TAA faz necessária a existência de um protocolo de implementação, bem como a existência de indicadores analíticos, fisiológicos, patológicos e comportamentais do animal em questão.

\subsection{Cinoterapia}

A Cinoterapia ou terapia assistida por cães (TAC), é uma das modalidades da terapia assistida por animais que utiliza o cão como co-terapeuta. Teve sua origem no século XVIII, na Inglaterra, quando foi descoberto que o convívio com cães trazia benefícios psicológicos, pedagógicos e sociais ao seu praticante. (OLIVEIRA, 2007, apud FISCHER et al., 2016). Sendo estabelecidos como base a motivação, sensibilidade, concentração e socialização, tanto do animal quanto do paciente. Os cães são os animais mais utilizados para as práticas de TAA devido a sua sociabilidade, fácil adestramento e maior aceitação por parte das pessoas (KOBAYASHI et al., 2009, apud FREITAS et al., 2018).

O vínculo estabelecido entre o animal e o paciente, faz com que este último se dedique a cultivar sentimentos de gentileza, estima, identificação de uma amizade, confiança e compaixão. Esse vínculo é indicado por Carvalho (2014, apud FERREIRA; GOMES, 2017) como mecanismo fundamental para os órgãos sensoriais, sistema límbico e sentido cinestético.

Estudos comprovam os benefícios de um cão na vida de um ser humano, o simples ato de acariciar o animal é capaz de reduzir a pressão arterial, liberando o hormônio do relaxamento, a serotonina, e diminuindo o hormônio do estresse, o cortisol (SOUSA, 2016). E, segundo Odendaal (2000, apud FISCHER et al., 2016), benefícios também podem ser identificados no cão, sendo eles: aumento de endorfina, ocitocina, prolactina, ácido fenilacético e a diminuição do cortisol e da pressão sanguínea.

Para Ailello (2005, apud SOUSA, 2016), a escolha do cão terapeuta deve ser feita considerando seu temperamento, socialização e adestramento. Todavia, apesar 
da raça permitir conhecer melhor o temperamento e comportamento do animal, esse não é um requisito de escolha. O principal fator é sem dúvidas o adestramento, onde os animais selecionados são avaliados por profissionais específicos, através de testes característicos.

A idade também deve ser levada em conta, pois cães muito jovens são mais inquietos, possuem garras e dentes afiados, enquanto cães muito velhos se cansam mais rapidamente. O que os torna inviáveis para uso em Cinoterapia. Fêmeas no cio também não devem ser utilizadas na prática, porque poderiam desviar a atenção nos machos presentes em seu entorno.

Os cães devem ser avaliados semestralmente por meio de hemogramas completos, além de apresentação de vacinação e vermifugação em dia. Também é essencial que eles estejam limpos e higienizados no momento da TAC.

\subsubsection{Equoterapia}

A equoterapia é um método terapêutico e educacional que,a partir do contato com o cavalo, pretende desenvolver o praticante em suas dimensões biológica, psicológica, social e espiritual (SEVERO, 2010, apud PAVÃO, 2015). Essa terapia é uma alternativa de tratamento não medicamentoso em que se trabalham as várias formas do desenvolvimento da criança, de forma lúdica, com o cavalo e em seu ambiente natural (SILVA, 2004, apud GATI, 2016). Mas não é uma prática exclusiva para crianças, ela auxilia pessoas portadoras de necessidades especiais motoras e mentais, e segundo Dotti (2014, apud FERREIRA; GOMES, 2017) ela também é indicada para pessoas "saudáveis" com problemas de postura, insônia e estresse.

Alguns autores, como Despret (2004, apud PAVÃO, 2015) a consideram como uma prática antropo-zoo-genética, defendendo que ao invés de uma interação entre homem e animal, ocorre uma intra-acão, visto que cavalo e o praticante sofrem alterações, não necessariamente simétrica para ambos.

Sendo uma modalidade de terapia assistida por animais (TAA), a equoterapia teve seus primeiros relatos como tratamento médico no século XVII, com o objetivo de melhorar o controle postural, a coordenação e o equilíbrio de pacientes com distúrbios articulares (DE PAUW, 1984, apud ECKERT, 2013).

No Brasil, somente em 1997 o tratamento foi reconhecido como um método terapêutico, pelo Conselho Federal de Medicina e pela Sociedade Brasileira de 
Medicina Física e Reabilitacional, de acordo com a legislação brasileira. Atualmente, a equoterapia compõe os serviços especializados oferecidos pelo Sistema Único de Saúde (SUS) (ANDE-BRASIL, 2007; KLEIN, 2007, apud GATI, 2016).

A seleção do cavalo adequado é fundamental para o desenvolvimento da equoterapia, visando a segurança física do praticante, que deve ser uma preocupação constante para toda a equipe. Um animal terapeuta deve demonstrar comportamento confiável, controlado, previsível e inspirar confiança naquele que está a interagir com ele (CAMPOS, 2009, apud GATI, 2016).

Para a escolha, há também uma classificação de acordo com o porte do animal e seu ritmo de andar.

O cavalo pode movimentar-se de três modos: ao passo, ao trote e ao galope. Em cada uma dessas modalidades seus movimentos são distintos, por isso há diferença de qual será usada na terapia, com dependência do tipo de pessoa que fará uso dela. Todas essas modificações de atitudes impõem um ajuste muscular ao praticante, a fim de responder aos desequilíbrios provocados (WICKERT, 1999, apud ECKERT, 2013).

Em suma, é necessário o conhecimento de toda a conformação do equino, ou seja, conhecimento em hipologia e etologia, para saber quanto cada cavalo pode contribuir na equoterapia. Busca-se, principalmente, realizar o treinamento também através de reforços positivos, sendo importante a aplicação da dessensibilização progressiva (base para testes comportamentais) a fim de que haja uma acomodação sensitiva e o animal não apresente significativos níveis de estresse diante de diferentes estímulos.

A duração da equoterapia também não pode ser longa, para evitar que o cavalo fique cansado, estressado e sobrecarregado ao longo do dia, evitando assim transtornos físicos e mentais caracterizantes da Síndrome de Exaustão Equina.

\subsection{Bem-estar $\mathrm{x}$ estresse}

O bem-estar é definido como o estado do indivíduo relacionado as suas tentativas de lidar satisfatoriamente com o seu ambiente (BROOM, 1986, apud ROCHA, 2015), diminuindo o sofrimento físico, comportamental e fisiológico e mantendo em harmonia as funções do corpo. Quando essa homeostasiado animal está em risco, seja por qual for o estímulo externo, levando a uma modificação no funcionamento do organismo, é que surge o estresse. Para Veissier \& Boissy (2006, 
apud ALMEIDA, 2015), essa modificação é que prepara o animal para futuros desafios.

Esse pensamento sugere que determinada situação estressante pode vir a se tornar insignificante para o animal, através da aplicação de técnicas como, por exemplo, a habituação. O que é muito importante no momento do adestramento dos animais. Propiciar novos estímulos, exercícios e sociabilização, inibem o surgimento de problemas comportamentais (GERGE e ROSSI, 2011, apud FISCHER, 2016), evitando uma possível situação de estresse e mantendo, portanto, o bem-estar do animal. Por isso o treinamento básico de obediência e concentração pode ser descrito como o primeiro passo para a garantia do bem-estar do animal utilizado em terapia.

Ressaltando que, no treinamento, de igual maneira é fundamental a priorização do bem-estar, zelando pelos direitos dos animais. Esse treinamento deve ser realizado pela aplicação de reforços positivos, descartando qualquer tipo de punição positiva ou de reforço negativo, a fim de se evitar o estresse e respostas negativas. É fundamental que o animal receba afeto em todas as etapas do treinamento e da terapia.

Diferentes autores relatam que a TAA não está isenta de expor o animal a situações estressantes. A intensidade das atividades pode levar ao aumento de cortisol, indicador de resposta ao estresse em estudos de bem-estar animal (COPOLA et al., 2006, apud ALMEIDA, 2015); inclusive o trabalho terapêutico em si pode ser estressante devido a diversos fatores como: presença de muitas pessoas ao redor, barulho, temperatura elevada, exigência em excesso do animal, determinados odores, exposição a estranhos, entre outros.

O estresse obtido pode ser avaliado por meio de variações hormonais, através de amostras de sangue, urina e fezes. O comportamento do animal também é capaz de indicar a exposição a alguma situação estressante, variando de acordo com a espécie.

Portanto, tendo em vista que a sociedade de um modo geral vem se mobilizando para a promoção do bem-estar (WHITE, 2009, apud FISCHER 2016), a TAA deve se moldar em uma base moral, priorizando os direitos dos animais no sentido de respeitar as cinco liberdades e propiciar a eles um ambiente adequado com experiências positivas e recíprocas. 


\section{Metodologia}

O presente trabalho interdisciplinar foi desenvolvido a partir de uma pesquisa exploratória com o objetivo de adquirir familiaridade e construir opiniões sobre o tema "Os possíveis estresses causados em animais utilizados em terapias: cinoterapia e equoterapia", se tratando de uma revisão bibliográfica. A revisão bibliográfica é elaborada a partir do uso de dados publicados anteriormente (GIL, 2002), investigando as influências desses dados na atualidade, ou mesmo os avanços e descobertas de determinado tema no tempo decorrido.

Inicialmente, para a composição do referencial teórico foi realizada uma leitura prévia de artigos, dissertações, publicações, teses e materiais encontrados principalmente nos bancos de dados virtuais: ScieLO e Google Acadêmico. Materiais relacionados à Terapia Assistida por Animais,com um foco maior no animal e no seu bem-estar, foram priorizados na escolha. Não descartando aqueles materiais com foco em humanos, pois quando comparados é possível se criar uma discussão em torno da terapia a partir de diferentes pontos de vista.

Complementarmente, foi realizada uma entrevista com o médico veterinário Gregory Rocha Almeida, formado da Faculdade Univertix (Matipo), responsável pela saúde do equino na equoterapia de Teófilo Otoni, a respeito do estresse na terapia assistida e de como evitá-lo, ressaltando as informações encontradas nos materiais bibliográficos utilizados.

Os diferentes autores permitiram a realização de um breve apanhado histórico em torno do tema, o que se consiste no embasamento para a análise dos dados obtidos em torno da existência ou não do estresse causado nos animais utilizados em terapias.

\section{Análise de dados}

Embora um dos artigos defenda que subjetivamente a terapia assistida por animais não cause qualquer estresse neles, todos os demais autores reconhecem que há sim essa possibilidade. São variadas as possíveis causas do estresse nesses animais, podendo ser oriundos de problemas que eles possam vir a ter com a socialização, os equipamentos existentes no local da terapia, o local em si, odores, comida, barulhos incomuns, objetos estranhos e temperatura. 
A intensidade da terapia também é apontada como um forte indício de aumento de cortisol, podendo vir a se tornar determinante do estresse crônico nesses animais. Portanto, a própria terapia pode ser causadora de estresse, quando não baseada nos direitos dos animais, mas em condutas éticas antropocêntricas.

Mais especificamente na cinoterapia, há casos de reforço negativo em treinamentos, excesso de trabalho, fadiga, ansiedade, limitação ao acesso de água e estresse calórico (BURROWS et al., 2008, apud FISCHER, 2016) presentes na terapia. Fatores que indicam um estresse do tipo físico e do tipo emocional (ansiedade), e podem levar o animal a desenvolver a Síndrome de Burnout. O simples deslocamento até o local da terapia e a exposição a novos ambientes, podem ser causas de estresse em cães. Um exemplo de ambiente estressor para esses animais é o hospital, quando há cheiros que podem ser agressivos ao olfato sensível dos cães, gerando desconforto.

Cães estressados podem apresentar problemas digestivos, dermatológicos e comportamentais (aumentar locomoção, levantar a pata, sacudir o corpo, bocejar e lamber os lábios). Além das comuns alterações fisiológicas em animais de um modo geral, tão comumente representadas pela fisiologia do estresse.

As causas de estresse são semelhantes na equoterapia. Equinos são expostos ao estresse diante de movimentos repetitivos (SOUZA et al., 2007, apud FORTES et al., 2014), da má alimentação, que não seja suficiente para repor suas energias, da exposição a estímulos inesperados, intensidade da terapia e treinamento inadequado que não faça uso da doma racional. Altas temperaturas, não acesso a água e ausência de sombras nas áreas de descanso provocam o estresse calórico no animal.

Cavalos não devem ser impedidos de realizar comportamentos inerentes, o que pode ser observado nas diferenças de comportamentos apresentados quando eles estão presos nas baias para quando estão soltos no pasto. Logo, deixá-los presos por muito é também uma causa de estresse, no qual esses animais apresentam comportamento mais apático.

Em ambos os casos, portanto, seria mais viável dizer que existem causas de estresse na terapia assistida por animais, não apenas que elas "podem" existir, por mais que o estresse seja mínimo, como apontado pelo médico veterinário Gregory Rocha. O animal está sujeito a novos estímulos todo o tempo, estando assim 
exposto ao aumento do cortisol e, consequentemente, alterações comportamentais e fisiológicas. Resta ao homem impedir que esse estresse se torne crônico.

Tanto os cães quanto os cavalos enfrentam situações que provocam 0 estresse físico e emocional em seus organismos. Dentre o estresse físico, pode-se dizer que o mais comum é o estresse calórico. Enquanto o estresse emocional é muito representado demonstrativamente pelo desencadeamento de sensações de medo, ansiedade e frustração.

Visto por esse ponto, a aplicação do bem-estar é notória quando se fala em terapia assistida por animais, como demonstrado pela maior utilização de uma ética bem-estarista nessa prática (58\%), em detrimento da ética antropocêntrica utilitarista (32\%) e da ética utilitarista permissiva (18\%) (FISCHER et al.,2016). O que demonstra a importância de falar a respeito dos possíveis estresses existentes nos animais utilizados em terapias, em busca da implantação de técnicas que o diminuam o máximo possível.

Dentre essas técnicas, a promoção de boas condições de manutenção, manipulação e transporte podem refletir em métodos de avaliação do grau de bemestar através de parâmetros de dor e sofrimento (SINGER, 2004, apud FISCHER et al., 2016). A questão do adestramento do animal pode ser considerada o ponto chave para minimizar situações de estresse, condicionando 0 animal a comportamentos adquiridos, com destaque para os métodos de habituação e de dessensibilização progressiva. A indução de testes comportamentais prévios são, portanto, um passo determinante.

É certo que é inevitável que o animal haja institivamente em determinados momentos, o que implica em um planejamento de imprevistos (movimentos involuntários do paciente, número de pacientes, temperatura do lugar, exigência física do animal, entre outros) causadores de estresse, a fim de minimizá-los. Seria como uma maneira de prever as variáveis da rotina do animal, tornando-as algo comum para eles.

Portanto, vale dizer que a terapia assistida por animais deve ser implantada lado a lado da bioética. A Bioética conduz a reflexão da TAA, ponderando custos, benefícios e alternativas, bem como estimulando a autonomia do paciente ou seu responsável legal. Para minimizar os efeitos das imprevisibilidades faz-se necessária 
à existência de um protocolo de implementação do programa. (FISCHER et al., 2016)

Ela é regida pelo princípio ético na tomada de decisões, com uma tríplice função descritiva, normativa e protetora.

\section{Considerações Finais}

Seja do ponto de vista bioético ou fora dele, foi observado que alguns autores possuem opiniões extremamente opostas quanto ao uso de animais em terapias. Alguns alegam que nessa situação os animais estão expostos a condições não naturais, sendo privados da maior parte do seu comportamento inato, apenas tolerando o ambiente a que são submetidos. Defendem que usar animais em terapias é uma prática inteiramente assimétrica.

Outros defendem uma visão antropocêntrica, encarando um animal como um simples recurso ou objeto usado em prol do benefício humano. Enquanto alguns defendem que os direitos dos animais são levados em conta na TAA, sendo eles (animais) vistos como resultado de uma relação simbiótica consolidada durante 0 processo evolutivo de ambos (homem e animal).

Esses pontos divergentes poderiam levar a temas de discussão e talvez despertar o interesse de profissionais da medicina veterinária, ou de outras áreas, a desenvolverem mais estudos a respeito do tema.

É inegável dizer que os animais não sofrem estresse na TAA, mas tem que se levar em conta que mesmo em seu ambiente natural eles estão sujeitos a apresentarem aumento de cortisol que culmine em alterações fisiológicas e comportamentais. Existem agentes estressores na TAA? Sim, existem, mas isso não quer dizer que eles não possam ser minimizados e se tornarem sem significância na rotina do animal utilizado nessa prática.

Para que isso seja possível, basta respeitar os direitos pertencentes ao animal e entender que ele não é um simples recurso da terapia. Isso pode ser feito respeitando seus processos biológicos, oferecendo afeto, supervisionando os treinamentos, as terapias em si e o mais importante, não naturalizando os maus tratos. Afinal, o animal é o verdadeiro terapeuta da TAA, não um meio para ela. $E$ os benefícios originados com a prática podem sim ser simétricos, traduzindo o reforço de um antepassado vinculo existente entre homem e animal. 


\section{Referências}

ALMEIDA. J. F., AGUIAR. V.M., PEDRO. D. A., Levantamento sobre a percepção das pessoas em relação à terapia assistida por animais. Revista Brasileira de Zoociências..16: pp.85 - 92. 2014/2015

ALMEIDA J. L. E. A., Comparação entre bem-estar psicológico do tutor e problemas comportamentais o seu animal de companhia. Dissertação para a obtenção do Grau de Mestre em Medicina Veterinária pela Universidade Lusófona de Humanidades e Tecnologias- Faculdade de Medicina Veterinária. Lisboa, 2015.

COSTA. M. P. D., GATO. F., RODRIGUES. N. M. Utilização de terapia assistida por animais como ferramenta no tratamento de doenças em humanos:

Revisão. v.12, n.1, a1, p.1-7, jan, 2018

ECKERT. D. Equoterapia como recurso terapêutico: análise eletromiográfica dos músculos reto do abdômen e paravertebral durante a montaria. Dissertação de Mestrado, apresentada ao Programa de Pós-Graduação em Ambiente e Desenvolvimento do Centro Universitário Univates como requisito para a obtenção do grau acadêmico de Mestre em Ambiente e Desenvolvimento, linha de pesquisa e Ecologia. Lajeado, Rio Grande do Sul. 2013.

FERREIRA. A. P. S., GOMES. J. B., Levantamento Histórico da Terapia Assistida por Animais. Revista Multidisciplinar PeyKëyo Científico. pp. 71-92. 2017. FISCHER, M. L., ZANATTA. A. A., REZENDE. E. A. Um olhar da bioética para a zooterapia. Una mirada de la bioética para lazooterapia. Revista Latinoamericana de Bioética, vol. 16, núm. 1, janeiro- junho, 2016, pp.174-197.

FORTES. C. H. M., NASCIMENTO. C. A., LINCK. I. M., PARENZONI. V.C. O bemestar animal e a equoterapia: Um gesto de leitura. XIX Seminário Interinstitucional de Ensino, Pesquisa e Extensão da Unicruz. 2014.

FREITAS. D. B. A., MISTIERI. M. L. A., SOARES. C. D., GOMES. E. M. Desenvolvimento de atividades assistida por cães e o papel do médico veterinário. Rev. Ciênc. Ext. v.14, n.3, p.22-30, 2018.

GATI. L. Equoterapia. Revista CFMV: Conselho Federal Medicina Veterinária. p.14-24.Brasília-DF. n 61.Jan/Abril 2014/2016. Ano XX

GIL, A. C. Como Elaborar Projetos de Pesquisa. 4. ed. São Paulo: Atlas, 2002.

HERRERA. C. C., FONSECA. M. S.TerapiasAsistidasconAnimales: Una Perspectiva de Protección Animal. Revista Scientific. - Ensayo Arbitrado. Vol. 3, № 9 - AgostoOctubre 2018 - pág. 275/296. Venezuela.

KOBAYASHI CT, et al. Desenvolvimento e implantação de Terapia Assistida por Animais em hospital universitário. Revista Brasileira de Enfermagem, vol. 62, núm. 4, julho-agosto, 2009, pp. 632-636. Brasília. 
LÔBO. J. F. A., Análise conformacional dos equinos utilizados na equoterapia do centro de reabilitação e readaptação Doutor Henrique Santillo, Goiânia, Goiás. Dissertação para obtenção do título de Mestre em Ciência Animal junto à Escola de Veterinária e Zootecnia da Universidade Federal de Goiás. 2016.

MACHADO, J.A.C et al. Terapia Assistida por Animais (TAA). Rev. Científica Eletrônica de Medicina Veterinária - ISSN: 1679-7353. Ano VI - Número 10 Janeiro de 2008 - Periódicos Semestrais.

MENEGAZZO. A. D., SOUZA. V. S. W., C. J. S., FIÓRIO. F. B., Influência da cinoterapia e perfil do animal durante exercícios fisioterapêuticos na Síndrome de Smith Lemli Optiza. FisiSenectus Unochapecó. Ano 3, n. 1 - Jan/Jun. 2015 p. 29-37.

PAVÃO. L. C., "“O que é que cavalo sabe”": Um estudo antropológico sobre o vínculo animal-humano na equoterapia. Dissertação de Pós-Graduação em Antropologia Social da Universidade Federal de São Carlos, para obtenção do título de Mestre em Antropologia. São Carlos- SP.2015

ROCHA. C. F. P. G., Avaliação comportamental e endócrina do nível de estresse de cães participantes de intervenções assistida por animais. Dissertação de Programa Pós-Graduação em Psicologia Experimental para obtenção do título em Mestre em Psicologia. Universidade de São Paulo, São Paulo. 2015

SILVA. J. da. M., Terapia Assistida por Animais. Tese para obtenção do título de monografia do curso Medicina Veterinária. Universidade Federal de Campina Grande- Centro de Saúde e Tecnologia Rural Campus de Patos-PB. 2010

SOARES. D. F. G., OTONE. G. A., FAícO. M. M., de M., Equoterapia Teoria e Prática. 1. ed. Caratinga. FUNEC Editora, 2011.

SOUZA. F. T. C., SOUSA. M.E.S., SANTOS, N.D. L., Gestão de resíduos sólidos: Uma alternativa para alimentação de animais ruminantes. Ciência Agrícola, Rio Largo. V 15. p. 45-48. 2017

SOUSA. N. K. L. Terapia facilitada por cães: Estudo de caso. Tese de trabalho de conclusão de curso no Centro de Ciências Agrárias da Universidade Federal da Paraíba, para obtenção do título de Bacharel em Zootecnia. Areia-PB. 2016.

WERNEQUE. K. C. T., Terapia medida por animais:histórico, áreas de aplicação e resultados terapêuticos. Universidade Vale do Rio Doce-UNIVALE. Faculdade de ciências humanas e socais-FHS. Govenador Valadares, 2004.

YAMAMOTO. K. C. M., SILVA. E. Y. T., COSTA. K. N., SOUZA. M. S., SILVA. M. L. M., ALBUQUERQUE. V. B., PINHEIRO. D. M., BERNABÉ. D. G., OLIVA. V. N. L. S., Avaliação fisiológica e comportamental de cães utilizados em terapia assistida por animais (TAA). Phyisiological and behavioral assessment in dogs used in Animal-Assisted Therapy (AAT). Arq. Bras. Med. Vet. Zootec., v.64, n.3, p.568-576, 2012. 